\title{
Cytochrome P450 monooxygenase genes in the wild silkworm, Bombyx mandarina
}

\author{
Linrong Wan Equal first author, 1,2 , Anlian Zhou ${ }^{\text {Equal first author, }{ }^{1} \text {, Wenfu Xiao }}{ }^{1}$, Bangxing Zou ${ }^{1}$, Yaming Jiang ${ }^{1}$, Jinshu Xiao ${ }^{1}$, Cao \\ Deng $^{\text {Corresp., } 3,4}$, Youhong Zhang ${ }^{\text {Corresp., }}{ }^{,}$, Genefang Research Team ${ }^{3}$ \\ ${ }^{1}$ Sericultural Research Institute, Sichuan Academy of Agricultural Sciences, Nanchong, Sichuan, China \\ 2 College of Agronomy, Sichuan Agricultural University, Chengdu, Sichuan, China \\ 3 Research and Development Center, Genefang, Chengdu, Sichuan, China \\ 4 Departments of Bioinformatics, DNA Stories Bioinformatics Center, Chengdu, Sichuan, China \\ Corresponding Authors: Cao Deng, Youhong Zhang \\ Email address: dengcao@genefang.com, sczhangyh@126.com
}

Wild (Bombyx mandarina) and domestic silkworms (Bombyx mori) are good models for investigating insect domestication, as 5000 years of artificial breeding and selection have resulted in significant differences between $B$. mandarina and $B$. mori. In this study, we improved the genome assemblies to the chromosome level and updated the proteincoding gene annotations for $B$. mandarina. Based on this updated genome, we identified 68 cytochrome P450 genes in $B$. mandarina. The cytochrome P450 repository in $B$. mandarina is smaller than in $B$. mori. Certain currently unknown key genes, rather than gene number, are critical for insecticide resistance in $B$. mandarina, which shows greater resistance to insecticides than $B$. mori. Based on the physical maps of $B$. mandarina, we located 66 cytochrome P450s on 18 different chromosomes, and 27 of the cytochrome P450 genes were concentrated into 7 clusters. KEGG enrichment analysis of the P450 genes revealed the involvement of cytochrome P450 genes in hormone biosynthesis. Analyses of the silk gland transcriptome identified candidate cytochrome P450 genes (CYP306A) involved in ecdysteroidogenesis and insecticide metabolism in B. mandarina. 


\section{Cytochrome P450 monooxygenase genes in the wild}

2 silkworm, Bombyx mandarina

3

Linrong Wan ${ }^{1,2, *}$, Anlian Zhou ${ }^{1,}$, Wenfu Xiao ${ }^{1}$, Bangxing Zou ${ }^{1}$,Yaming Jiang ${ }^{1}$, Jinshu Xiao ${ }^{1}$, Cao Deng ${ }^{3,4}$ and Youhong Zhang ${ }^{1}$, Genefang Research Team ${ }^{3}$

${ }^{1}$ Sericultural Research Institute, Sichuan Academy of Agricultural Sciences, Nanchong, Sichuan, China

${ }^{2}$ College of Agronomy, Sichuan Agricultural University, Wenjiang, Chengdu, Sichuan, China

${ }^{3}$ Research and Development Center, Genefang, Chengdu, Sichuan, China

${ }^{4}$ Departments of Bioinformatics, DNA Stories Bioinformatics Center, Chengdu, Sichuan, China

* These authors contributed equally to this work.

Corresponding Authors:

Youhong Zhang, sczhangyh@126.com

Cao Deng, dengcao@genefang.com

\section{Abstract}

Wild (Bombyx mandarina) and domestic silkworms (Bombyx mori) are good models for investigating insect domestication, as 5000 years of artificial breeding and selection have resulted in significant differences between B. mandarina and B. mori. In this study, we improved the genome assemblies to the chromosome level and updated the protein-coding gene annotations for $B$. mandarina. Based on this updated genome, we identified 68 cytochrome P450 genes in B. mandarina. The cytochrome $\mathrm{P} 450$ repository in B. mandarina is smaller than in $B$. mori. Certain currently unknown key genes, rather than gene number, are critical for insecticide resistance in B. mandarina, which shows greater resistance to insecticides than B. mori. Based on the physical maps of B. mandarina, we located 66 cytochrome P450s on 18 different chromosomes, and 27 of the cytochrome P450 genes were concentrated into 7 clusters. KEGG enrichment analysis of the P450 genes revealed the involvement of cytochrome P450 genes in hormone biosynthesis. Analyses of the silk gland transcriptome identified candidate cytochrome P450 genes (CYP306A) involved in ecdysteroidogenesis and insecticide metabolism in $B$. mandarina.

\section{Key words}

Bombyx mandarina, wild silkworm, cytochrome P450, gene family. 
35

\section{Introduction}

The domestic silkworm, Bombyx mori, is a model insect often used to study physiology, biochemistry, developmental biology, neurobiology, and pathology (Kawamoto et al. 2019). It has been reared for more than 5000 years for silk production (Fang et al. 2015), and it is now used for commercial production of medically and industrially important biomaterials based on genetic engineering. The wild silkworm (Bombyx mandarina), the direct ancestor of $B$. mori, is a valuable gene pool resource that can be exploited and utilized. B. mandarina provides an important basic material to study the origin and differentiation of silkworms and is significant for B. mori breeding and the establishment and application of a gene pool for special traits.

A draft sequence of the B. mori genome was first reported by Chinese and Japanese groups (Xia et al. 2004), and the annotation of both WGS data sets with 8.48x sequence coverage was completed in 2008 (International Silkworm Genome 2008). Since then, many transcriptomic and evolutionary studies have investigated important bio-systems differing between the domestic and wild silkworms based on the genome sequence. Kawamoto et al. performed hybrid assembly of B. mori based on $140 \times$ deep sequencing of long (PacBio) and short (Illumina) reads and annotated the new genome with more RNASeq and protein data, generating higher quality genome assemblies and more accurate gene models (Kawamoto et al. 2019). To reconstruct the domestication processes and to identify selective sweeps among $B$. mori strains, Xiang et al. generated a draft assembly for $B$. mandarina using a classic shotgun approach based on Illumina sequencing platforms (Xiang et al. 2018). These high-throughput datasets provide a more comprehensive way to study the similarities and differences between $B$. mori and B. mandarina at the whole genome level.

The cytochrome P450 monooxygenases (P450s) genes are a large and complex gene superfamily of heme-thiolate proteins. They are found in most organisms from prokaryotes to eukaryotes (Nelson 1999). The cytochrome P450 genes are an ancient enzymatic system, and all of the current cytochrome P450s may have descended from a common ancestral gene (Ai et al. 2011; Yu et al. 2015). P450 genes are responsible for the oxidative metabolism of structurally diverse endogenous and exogenous compounds (Nebert \& Gonzalez 1987). In insects, the cytochrome P450 enzymes catalyze the metabolism of physiologically important endogenous compounds, including hormones and pheromones at juvenile and molting stages, and they are well known for their detoxification in pesticides (Scott 1999; Scott et al. 1998). For this reason, many cytochrome P450s have been studied in insects, including Drosophila melanogaster, Anopheles gambiae, Aedes aegypti, Tribolium castaneum, Apis mellifera, and B. mori.

Based on the silkworm draft genome, Ai et al. identified 84 CYP-related sequences, which were classified into 26 families and 47 subfamilies according to the standard nomenclature (Ai et al. 2011). However, Kawamoto et al. identified a total of 83 cytochrome P450 genes using a 
high-quality genome assembly (Kawamoto et al. 2019). Considering the important role of $B$. mandarina for $B$. mori breeding and the important roles of cytochrome P450s in silkworms, it is interesting to explore the cytochrome $\mathrm{P} 450$ genes in $B$. mandarina and compare them to those in B. mori. In this study, the cytochrome P450 genes in B. mandarina were identified and a comprehensive genome-wide comparative analysis of the cytochrome P450 genes in $B$. mandarina and $B$. mori was performed. We linked the $B$. mandarina scaffold sequences generated by Xiang (Xiang et al. 2018) into pseudo-molecules using their syntenic to chromosomes in the B. mori. This study illuminates the functional diversities and the evolutionary mechanisms and significance of cytochrome P450s in both B. mori and $B$. mandarina.

\section{Materials \& Methods}

\section{Genome and annotation data resources}

Genomic and annotation data for B. mori were downloaded from SilkBase (http://silkbase.ab.a.u-tokyo.ac.jp/cgi-bin/download.cgi). This genome was published in 2019 and based on 140× deep sequencing of long (PacBio) and short (Illumina) reads. The new genome was annotated with more RNASeq and protein data, generating higher quality genome assemblies and more accurate gene models than the previous version (Kawamoto et al. 2019). Genomic and annotation data for B. mandarina were downloaded from the NCBI RefSeq (Accession: GCF_003987935.1, published in 2019) and assembled using a classic whole genome shotgun approach, based on Illumina sequencing platforms (Xiang et al. 2018). Genome and annotation datasets for other insect species are shown in Table S1.

\section{Improving the quality of the $B$. mandarina genome assembly}

To build chromosomes from genome contigs or scaffolds, we used an alignment-based approach. We used Lastz (Harris 2007) to perform the whole genome alignment between $B$. mandarina (Xiang et al. 2018) and B. mori (Kawamoto et al. 2019). Then, using the syntenic alignments between $B$. mandarina contigs and scaffolds and $B$. mori chromosomes, we recorded the strands and turns of $B$. mandarina contigs and scaffolds relative to the $B$. mori chromosomes. Finally, the wild and domestic contigs and scaffolds were linked into pseudo-molecules according to the strands and turns information (Table S2).

\section{Updating the gene annotation of $B$. mandarina}

The original B. mandarina NCBI RefSeq protein-coding gene models were generated by the automated NCBI Eukaryotic Genome Annotation Pipeline (https://www.ncbi.nlm.nih.gov/genome/annotation euk/Bombyx mandarina/100/). We first removed the low-quality protein-coding genes with coding sequences (CDS) shorter than $150 \mathrm{bp}$ and with stop codons in the CDSs. For the protein-coding gene models with alternative splicing, we only kept the longest CDS for each gene to generate a clean RefSeq annotation file in GFF3 
107

108

109

110

111

112

113

114

115

116

117

118

119

120

121

122

123

124

125

126

127

128

129

130

131

132

133

134

135

136

137

138

139

140

141

142

format.

Transcriptome sequencing raw data were downloaded from previous studies (Table S3). To obtain high-quality clean reads, the raw sequencing reads were filtered using Trimmomatic software (Bolger et al. 2014) with the following steps. First, reads with adaptor sequences were removed. Then, reads containing more than $20 \%$ of low-quality bases $(\mathrm{Q}<20)$ or containing more than $3 \%$ of ambiguous ' $N$ ' were discarded. The reads were also trimmed where the fourbases-window had an average quality lower than 20 . After these filtering steps, the clean reads from each sample were aligned to the updated genome assemblies using HiSAT2 (version 2.0.4) (Kim et al. 2015), which generated BAM files for downstream analyses.

The transcripts in each sample were assembled and merged using StringTie (version 1.3.1c) (Pertea et al. 2015) and the BAM files were generated by the HiSAT2 (Kim et al. 2015). If none of the transcripts in a gene model had overlaps with the RefSeq protein-coding gene models, the transcripts in this gene were subjected to TransDecoder (version r20140704) (2014) to predict the potential coding sequences (CDSs). This gene model was defined as a novel protein-coding gene model if it met the following requirements: (1) the CDSs had at least 50 codons; and (2) the CDSs had start- and stop-codons. We only retained the longest CDS for each novel proteincoding gene model.

To determine the functional annotation of the protein-coding gene models, a BLASTP (Camacho et al. 2009) search with an E-value $\leq 1 \mathrm{e}^{-5}$ was performed against protein databases, including NR (non-redundant protein sequences in NCBI) and SwissProt (Boeckmann et al. 2003). KEGG annotation that maps the B. mandarina cytochrome $\mathrm{P} 450$ genes to possible KO numbers and map numbers was fetched from KOBAS (version 3.0) (Xie et al. 2011) results. The domains and GO terms of each gene model were predicted by InterProScan (Quevillon et al. 2005) against public protein databases, including ProDom (Bru et al. 2005), PRINTS (Attwood et al. 1994), Pfam (Bateman et al. 2004), SMART (Ponting et al. 1999), PANTHER (Mi et al. 2005), PROSITE (Hulo et al. 2006), and TIGR (Haft et al. 2003).

\section{Synteny analysis of $B$. mandarina and $B$. mori}

To explore the collinearity of B. mandarina and B.mori, the updated B. mandarina proteome was blasted against to the B. mori proteome, and the MCScanX (Wang et al. 2012) was used to detect syntenic genes and blocks (regions with at least eight collinear genes). The collinearity of genes between B. mandarina and B. mori was visualized using Circos (Krzywinski et al. 2009).

\section{In silico identification of cytochrome $\mathbf{P 4 5 0}$ genes in $B$. mandarina and other insects}

The cytochrome P450 genes of $B$. mandarina were identified as following steps. First, we collected total 84 cytochrome P450 genes in B. mori, which included 82 cytochrome P450 genes from Kawamoto et al. (Kawamoto et al. 2019) and another two cytochrome P450 genes identified by Ai et al. but missing in Kawamoto et al. (Ai et al. 2011). The proteins and CDSs 
were extracted for downstream analyses. We also downloaded 91 P450 proteins from Drosophila melanogaster (http://drnelson.uthsc.edu/cytochromeP450.html), and then the cytochrome P450 proteins from $D$. melanogaster and B. mori were combined to form a reference cytochrome $\mathrm{P} 450$ protein database. Second, the $B$. mandarina proteome was searched against the reference cytochrome P450 protein database using the BLASTP algorithm (Camacho et al. 2009), with an e-value cut-off of 1e-5. For each B. mandarina protein, the top hit to the reference cytochrome $\mathrm{P} 450$ protein database was remained, and the $B$. mandarina proteins with hits to the reference cytochrome $\mathrm{P} 450$ proteins were the candidate cytochrome $\mathrm{P} 450$ proteins. Third, the domains of the $B$. mandarina cytochrome $\mathrm{P} 450$ candidates were annotated by the iprscan (version 5) (Quevillon et al. 2005) using the Pfam database (http://pfam.sanger.ac.uk/) (Bateman et al. 2004), and the candidate was identified as the cytochrome P450 gene if it had the PF00067 domain (cytochrome P450). Additional TBLASTN (Camacho et al. 2009)searches against the $B$. mandarina genomic assemblies followed by gene structure refinement using GeneWise (Birney et al. 2004) were also performed with the reference cytochrome $\mathrm{P} 450$ protein database to avoid missing cytochrome P450-related genes. We further manually corrected annotation for three genes (XP_028036989.1, XP_028036546.1 and XP_028040841.1) that were mis-predicted to generate artificial fusion genes by the NCBI Eukaryotic Genome Annotation Pipeline, and for these loci, we applied these criteria to define genuine protein-coding genes: (1) insertions, deletions or frameshifts were not allowed when they were compared to their homologous protein; (2) start codon and stop codon were compulsive, and (3) they had at least 50 amino acids.

The cytochrome P450 genes in other insect species (Table S1) were identified using the same pipeline. The exons and domains positions of silkworm cytochrome $\mathrm{P} 450$ genes were extracted from GFF3 file and Iprscan results, respectively, and then were plotted using the iTOL (interactive Tree Of Life, https://itol.embl.de/) (Letunic \& Bork 2019). The chromosomal locations for cytochrome P450 genes were plotted using karyoploteR (Gel \& Serra 2017). Phylogenetic analyses and classification of $B$. mandarina cytochrome $\mathbf{P 4 5 0}$ genes

The cytochrome P450 proteins were aligned using MUSCLE v3.8.31(Edgar 2004) with default parameters. RAxML (version 8.2.7) (Stamatakis 2014) was used to generate maximum likelihood with PROTGAMMALGX model and 100 bootstraps. Trees were plotted by the iTOL (https://itol.embl.de/)(Letunic \& Bork 2019). The phylogenetic tree between B. mandarina and B. mor $i$ and the standard nomenclature of $B$. mori were used to classify and name the $B$. mandarina cytochrome P450 genes (Ai et al. 2011; Kawamoto et al. 2019).

\section{RNA-Seq expression analysis of cytochrome $\mathbf{P 4 5 0}$ genes}

The gene expression levels of each sample were quantified using HTSeq (version 0.9.1) (Anders et al. 2014) and the BAM files generated by the HiSAT2 (Kim et al. 2015). Then, the DEGs between different sample pairs were detected via the edgeR software package (Robinson 
179

180

181

182

183

184

185

186

187

188

189

190

191

192

193

194

195

196

197

198

199

200

201

202

203

204

205

206

207

208

209

210

211

212

213

214

et al. 2010) of the R language. The P-values were corrected for false discovery rate (FDR) using multiple tests. Differentially expressed genes (DEGs) met the following criteria: FDR (adjusted P) $<0.05$ and $\mid \log 2$ fold change $(\mathrm{FC}) \mid>0.5$.

\section{GO and KEGG enrichment analysis}

Significantly over-represented GO terms among the $B$. mandarina cytochrome P450 genes were identified using the topGO package (Alexa \& Rahnenfuhrer 2010) in R programming language (https://www.r-project.org/). The enrichment of KEGG pathways was conducted with Fisher's exact test using R scripts. The significantly over-represented GO terms and KEGG maps were identified with adjusted $p$-values $\leq 0.05$.

\section{Results}

\section{Improvement of genome assembly to the chromosome level}

The draft assembly for $B$. mandarina described by Xiang et al. was fragmental and low quality (Xiang et al. 2018). There are a total of 3,105 scaffolds with a total length of 398,588,931 bp (395,983,407 bp without gaps). The scaffold and contig N50 were 2,789,315 bp and 29,637 $\mathrm{bp}$, respectively. The minimum sequence length was $500 \mathrm{bp}$, and the maximum sequence length was 14,129,094 bp. To improve the quality of genome assembly for B. mandarina, we implemented a reference-assisted approach to build chromosomes from genome contigs or scaffolds published by Xiang et al. (Xiang et al. 2018). A total of 337 scaffolds were linked into 28 chromosomes, and the total length of these chromosomes reached $98.35 \%(392,034,257 \mathrm{bp})$ of the raw assemblies published by Xiang et al. (Xiang et al. 2018) (Table S2). The shortest chromosome was chr2 (7,258,460 bp), while the longest one was chr24 (22,989,029 bp). Chr12 had the smallest size variation among B. mandarina and B. mori, while chromosome 4 had the largest size variation. To study the synteny of the two silkworms, the collinearity of genes between B. mandarina and B. mori was visualized using Circos (Fig. 1).

\section{Identification of novel protein-coding genes and gene function annotation}

To obtain more protein-coding gene models in the $B$. mandarina genome, we updated the gene annotation using StringTie-TransDecoder pipeline. Using these NCBI RefSeq representative protein-coding gene models as a reference, the StringTie assembled a total of 26,061 gene models with 41,390 transcripts. Among them, 14,005 gene models containing 29,053 novel transcripts (53.74\% of total gene models) had no overlaps with the NCBI RefSeq representative protein-coding gene models, and were defined as candidate novel genes. The candidate novel gene sequences were scanned directly for CDSs with TransDecoder (2014), which generated 1,939 novel protein-coding gene models after filtering out low-quality CDSs (see filtering criteria in the Methods). Together with a manually annotated cytochrome P450 gene (see next section), we identified 1,940 novel protein-coding gene models in the $B$. mandarina genome. When incorporating the NCBI RefSeq annotation, there were 14,212 
215

216

217

218

219

220

221

222

223

224

225

226

227

228

229

230

231

232

233

234

235

236

237

238

239

240

241

242

243

244

245

246

247

248

249

250

protein-coding gene models for the B. mandarina genome.

Among these 1,940 novel protein-coding genes, 1,295 genes (66.75\%) could be functionally annotated to at least one of the four databases, including NR (non-redundant protein sequences in NCBI), SwissProt (Boeckmann et al. 2003), KEGG, and GO. For the whole B. mandarina proteome, approximately $94.38 \%$ of genes could be functionally annotated compared with the $95.14 \%$ in B. mori.

\section{The identification and classification of cytochrome $\mathbf{P 4 5 0}$ genes in $B$. mandarina}

By integrating the results from homologous to $B$. mori cytochrome $\mathrm{P} 450$ proteins and the existence of complete cytochrome P450 domain (PF00067), we identified 67 cytochrome P450 genes in the $B$. mandarina genome. To find all of the cytochrome P450-related genes in the genomes, we further manually annotated the genome using the B. mori cytochrome P450 proteins as a reference (see Methods section). This homology-based gene annotation strategy detected one additional P450 gene, resulting in a total of 68 cytochrome P450 genes in the $B$. mandarina genome. For B. mori, Ai et al. identified 84 cytochrome P450 sequences based on a silkworm draft genome (Ai et al. 2011), while Kawamoto et al. identified 83 cytochrome P450 genes with a high-quality genome assembly (Kawamoto et al. 2019) and a unified set containing 84 cytochrome P450 genes was used in our downstream analyses (Table 1).

Although B. mandarina has a significantly smaller cytochrome $\mathrm{P} 450$ repository than B. mori, we did find two duplicated genes in the B. mandarina. These genes are all belong to CYP6AE subfamily members. Interestingly, in the cotton bollworm Helicoverpa armigera, CYP6AE gene cluster knockout using the CRISPR-Cas9-based genome editing tools reveals their roles in detoxification of phytochemicals and insecticides (Wang et al. 2018). Therefore, the duplicated CYP6AE subfamily members in the $B$. mandarina may contribute to their reduced susceptibility to the insecticides used for control (Bing et al. 2010).

We constructed a maximum likelihood tree using the $\mathrm{P} 450$ proteins from $B$. mandarina and B. mori (Fig. 2). Consistent with previous results, these $\mathrm{P} 450$ genes can be grouped into four major clades, which are common in the insects and include the CYP2, CYP3, CYP4 and mitochondrial CYP clades. Using the phylogenetic tree, the $68 \mathrm{P} 450$ genes in B. mandarina were classified into 25 families and 45 subfamilies according to the standard nomenclature and classification of P450 genes in B. mori. When compared with B. mori, the CYP365 family is missing in B. mandarina. For the two cytochrome P450 genes in B. mori identified by Ai et al. but missing in the Kawamoto et al. (Ai et al. 2011), the CYP6AV1 is present in B. mandarina, but the CYP341A6 is missing.

The structural divergence of gene family members may arise due to exon/intron loss or gain and other mechanisms, and analyses of exon/intron structures can be important in revealing the evolutionary history of the gene family. The investigation of the intron-exon organizations of $B$. 
251

252

253

254

255

256

257

258

259

260

261

262

263

264

265

266

267

268

269

270

271

272

273

274

275

276

277

278

279

280

281

282

283

284

285

286

mandarina cytochrome P450 genes revealed highly variable intron-exon structures among these genes (Fig. S1). However, the genes in a clade of the CYP3 clan, which is the largest clan and is most closely related to vertebrate CYP3 and CYP5 families, have significantly smaller numbers of exons and a longer length for each exon than those in the other clans. These similarities in exon-intron organization in this clade provide a strong support for a common origin.

\section{Genomic distribution of $\mathbf{P 4 5 0}$ superfamily in the silkworm}

Based on the physical maps, we located 66 cytochrome P450s on 18 different chromosomes in B. mandarina. Only two genes were on the scaffolds. The XP_028042624.1 (CYP9A21) was on the scaffold NW_021012135.1, while its B. mori ortholog was on chromosome 17. The XP_028042717.1 (CYP4S5) was on scaffold NW_021013128.1, while its ortholog in the B. mori was on chromosome 21.

The genomic distribution patterns of the cytochrome P450 genes in these two silkworm species are different. For B. mandarina, 27 cytochrome P450 genes were concentrated into 7 clusters, which are defined as containing at least three genes, while for B. mori, 37 cytochrome P450 genes were concentrated into 8 clusters (Fig. 3). B. mori has one more cluster, which is located on chromosome 21 and has four genes. Three of the four genes were not found in $B$. mandarina, indicating potential loss. The largest single cluster in B. mori is located on chromosome 26, which has 9 CYP340 genes; however, the synteny regions in B. mandarina only have 4 CYP340 genes.

Figure 3 shows that these two species also share common characteristics in the genomic distribution of cytochrome P450 genes. The P450s are unevenly distributed in the genome. Most of the cytochrome P450s are tandemly arranged on chromosomes in both silkworm species. All of the CYP340 genes are located on chromosome 26 and form at least two clusters. All of the CYP341 genes are located on chromosome 22, and form a cluster and a singleton. CYP9 genes are located in the chromosome 17 and form a cluster, with the exception of CYP9G1, which is located on chromosome 15 as a singleton.

\section{Computational functional analyses of $B$. mandarina cytochrome $\mathbf{P 4 5 0}$ genes}

To explore the functional roles of the cytochrome P450 genes in B. mandarina, we obtained the GO terms from Gene Ontology (GO), which classifies genes into three GO categories: cellular component, molecular function, and biological process. Using the topGO (Alexa \& Rahnenfuhrer 2010) package in R programming language (https://www.r-project.org/), we identified four over-represented GO terms for molecular function and one over-represented term for biological process (Table S6). For the molecular function, there were 67 of 107 cytochrome P450 genes annotated to the GO:0020037 (heme binding, adjusted p-value: 0) and 3 of 16 cytochrome P450 genes annotated to the GO:0004497 (monooxygenase activity, adjusted pvalue $=0.045$; Table S6). These results are consistent with the fact that the cytochrome P450 
287

288

289

290

291

292

293

294

295

296

297

298

299

300

301

302

303

304

305

306

307

308

309

310

311

312

313

314

315

316

317

318

319

320

321

322

genes are heme-containing monooxygenases.

KEGG pathway-based analysis was also performed to determine the biological functions of cytochrome P450 genes in B. mandarina. A total of 63 cytochrome P450 genes $(92.65 \%$ of total cytochrome $\mathrm{P} 450$ genes) could be assigned to 13 non-redundant KO numbers using the KOBAS (Table S7). Among these KO orthologs, the five most abundant KOs were K14999 (gene number: 20, cytochrome P450 family 6 [EC:1.14.-.-]) and K15001 (gene number: 19, cytochrome P450 family 4 [EC:1.14.---]). Using KEGG mapper, 7 of these 13 KOs were mapped to the map00981 (Insect hormone biosynthesis), and in our KEGG enrichment analysis, this pathway was also significantly enriched with an adjusted p value of 4.48e-18 (Fig. 4). These results revealed the involvement of cytochrome P450 genes in insect hormone biosynthesis. Expression pattern of cytochrome $\mathbf{P 4 5 0}$ genes in silkworms

To explore cytochrome P450 gene expression in the silk gland, we collected two RNASeq datasets from silk gland, including the anterior silk gland (ASG), anterior median silk gland (AMSG), middle MSG (MMSG), posterior MSG (PMSG) and posterior silk gland (PSG) (Chang et al. 2015; Cheng et al. 2015). A total of 43 (63\% of 68) and 76 (90\% of 84) cytochrome P450 genes were expressed (raw counts $>=2$ ) in at least one tissue of B. mandarina and B. mori, respectively. Totally, we identified 10 differentially expressed genes (DEGs) and 32 DEGs in $B$. mandarina and B. mori tissues respectively (Fig. 5).

\section{Discussion}

By implementing a reference-assisted approach, we built chromosomes from genome contigs or scaffolds published by Xiang et al. (Xiang et al. 2018) for B. mandarina. The results revealed high genome synteny but also abundant structural rearrangements among the silkworms (Fig. 1). Despite building from a straight-forward alignment-based approach, our referenceassisted chromosome level assemblies can be used in downstream comparative genomic analysis and other types of analyses. We also updated the gene annotation using StringTie-TransDecoder pipeline, and this pipeline identified 1,940 novel protein-coding gene models in the $B$.

mandarina genome, indicating the necessity of updating the gene annotation.

We identified 67 cytochrome P450 genes in the B. mandarina genome (Table 1). The genomic distribution patterns of the cytochrome P450 genes in these two silkworm species are different (Fig. 3). Computational functional analyses of B. mandarina cytochrome P450 genes revealed the involvement of cytochrome P450 genes in insect hormone biosynthesis (Fig. 4). The P450 repository in B. mandarina is smaller than in B. mori and other insects, except for Vanessa tameamea (Table S5). This suggests loss of cytochrome P450 genes during evolution. Although B. mandarina is very similar to B. mori in physiological and morphological characteristics, resistance to insecticides differs between the two species due to natural selection. B. mori are weakly resistant to insecticides, and silk production is reduced by $>30 \%$ annually in China 
323

324

325

326

327

328

329

330

331

332

333

334

335

336

337

338

339

340

341

342

343

344

345

346

347

348

349

350

351

352

353

354

355

356

357

358

because of insecticide poisoning. In contrast, $B$. mandarina is a major pest of mulberry and are showing reduced susceptibility to the insecticides used for control (Bing et al. 2010). Cytochrome P450 genes play a major role in insecticide resistance, allowing faster metabolic removal of insecticides (Feyereisen 2015). However, the number of P450 genes in B. mandarina is much lower than that in B. mori, suggesting that selected key genes, rather than the total gene number of cytochrome P450s, are related to the increased resistance to insecticide resistance in $B$. mandarina.

Silk production is very different in B. mandarina and B. mori (Chang et al. 2015), and this phenotype attracted many attentions and accumulated many sequencing datasets (Chang et al. 2015; Cheng et al. 2015). The cytochrome P450 enzymes are found in almost all insect tissues. They fulfill many important tasks, from the synthesis and degradation of ecdysteroids and JHs to insecticide metabolism (Feyereisen 1999). Therefore, it is important to study the expression of cytochrome $\mathrm{P} 450$ genes in the silk glands of $B$. mandarina and B. mori. The silk gland is the only organ that produces silk proteins (fibroins and sericins). The silk gland is divided into three main parts: anterior silk gland (ASG), median silk gland (MSG), and posterior silk gland (PSG). The MSG can be further divided into anterior, middle, and posterior MSG (AMSG, MMSG, and PMSG, respectively) (Chang et al. 2015). PSGs are responsible for the synthesis and secretion of fibroins, MSG synthesize the sericins (glue proteins), and the ASG processes the liquid silk proteins and secretes them during cocoon formation (Fang et al. 2015). Two RNASeq datasets from the above tissues (Chang et al. 2015; Cheng et al. 2015) were used to explore cytochrome P450 gene expression in the silk gland.

The silk gland is greatly affected by insect hormones, especially, ecdysone and juvenile hormone $(\mathrm{JH})$. Growth and differentiations of the silk gland cells are accelerated by ecdysone, and are controlled by JH (Akai 1979). Daimon et al. revealed the essential role of CYP15C1 for the $\mathrm{JH}$ biosynthesis, and found that this gene is specifically expressed in the corpus allatum, an endocrine organ that synthesizes and secretes JHs (Daimon et al. 2012). However, we found that this gene is also expressed in the silk gland, although at a very low expression level (Fig. 5). Cheng et al. found four cytochrome P450 genes involved in ecdysteroidogenesis, including CYP306A1, CYP302A1, CYP315A1, and CYP314A1 (Cheng Dao-Jun 2014). Among these four genes, the CYP306A1 was found significantly differently expressed in both $B$. mandarina (XP_028040956.1) and B. mori (KWMTBOMO05794). CYP302A1 was only found to be significantly differently expressed in B. mori (KWMTBOMO13168) but not in B. mandarina (XP_028032374.1), while the CYP315A1 (KWMTBOMO04611 for B. mori, XP_028033300.1 for B. mandarina), and CYP314A1 (KWMTBOMO03959-03960M for B. mori, XP_028030999.1 for B. mandarina) were not differently expressed in the two silkworms (Fig. 5). Phoxim exposure is toxic to silkworms, causes a decrease of fibroin synthesis, and affects 
359

360

361

362

363

364

365

366

367

368

369

370

371

372

373

374

375

376

377

378

379

380

381

382

383

384

385

386

387

388

389

390

391

392

393

394

395

silk production. After phoxim exposure, Cheng et al. (Cheng et al. 2018) found that the transcriptional levels of $C Y P 6 A B$ and $C Y P 306 A$ were up-regulated by 1.731- and 1.221-fold, respectively. There are two and three CYP6AB genes in B. mandarina (XP_028030488.1, MSTRG.11650.2) and B. mori (KWMTBOMO06852, KWMTBOMO12342, and KWMTBOMO12343), respectively; however, they are not significantly expressed. Interestingly, CYP306A was found to be significantly differently expressed in both B. mandarina (XP_028040956.1) and B. mori (KWMTBOMO05794) (Fig. 5). We also checked the expression of CYP306A in the midgut from the B. mandarina, which tissues is one of the major tissues for insecticides metabolization. We blasted the CYP306A sequences to the RNASeq assemblies in the SilkBase (Kawamoto 2017), and the expression was validated by its homologous sequences in the SilkBase (A_BomaMG_comp25068_c0_seq1 and A_BomaMG_comp25068_c0_seq2).

\section{Conclusions}

In this study, we improved the quality of the genome assemblies and updated the proteincoding gene annotations for $B$. mandarina using the genome of $B$. mor $i$ as reference. Using in silico analyses of $B$. mandarina genomes, we identified 68 cytochrome $\mathrm{P} 450$ genes. Comparison with other insects revealed that $B$. mandarina may have lost many cytochrome P450 genes. Analyses of the silk gland transcriptome identified candidate cytochrome $\mathrm{P} 450$ genes (such as CYP306A) involved in ecdysteroidogenesis and insecticide metabolism in B. mandarina. Altogether, these results provided a genome-wide glimpse of the $B$. mandarina cytochrome P450 repository; however, the up- or down-regulated cytochrome P450genes require more wet experiments to explore their biological roles.

\section{Acknowledgements}

We would like to thank previous researchers and communities to submit the transcriptome data of the wild and domestic silkworm to NCBI SRA databases. We are grateful to the editor and anonymous reviewers for their comments and suggestions that have improved our manuscript greatly. The members of the Genefang Research Team: Zi-yan Huang, Cong-fan Bu, Jie Zeng, Zhao-nan Hao, Yan-peng Chen, and Meng-jia Liu.

\section{References}

Ai J, Zhu Y, Duan J, Yu Q, Zhang G, Wan F, and Xiang ZH. 2011. Genome-wide analysis of cytochrome P450 monooxygenase genes in the silkworm, Bombyx mori. Gene 480:42-50. Akai H. 1979. Hormonal Control of Silk Production in Silkworm, Bombyx mori. JARQ 13:116122.

Alexa A, and Rahnenfuhrer J. 2010. topGO: enrichment analysis for gene ontology. $R$ package version 2.

Anders S, Pyl PT, and Huber W. 2014. HTSeq — a Python framework to work with highthroughput sequencing data. Bioinformatics 31:166-169.

Attwood T, Beck M, Bleasby A, and Parry-Smith D. 1994. PRINTS--a database of protein motif 
396

397

398

399

400

401

402

403

404

405

406

407

408

409

410

411

412

413

414

415

416

417

418

419

420

421

422

423

424

425

426

427

428

429

430

431

432

433

434

435

436

fingerprints. Nucleic Acids Research 22:3590.

Bateman A, Coin L, Durbin R, Finn RD, Hollich V, Griffiths-Jones S, Khanna A, Marshall M, Moxon S, and Sonnhammer EL. 2004. The Pfam protein families database. Nucleic Acids Research 32:D138-D141.

Bing L, Yanhong W, Haitao L, YaXiang X, Zhengguo W, YuHua C, and Weide S. 2010. Resistance comparison of domesticated silkworm (Bombyx mori L.) and wild silkworm (Bombyx mandarina M.) to phoxim insecticide. African Journal of Biotechnology 9:1771-1775.

Birney E, Clamp M, and Durbin R. 2004. GeneWise and genomewise. Genome Research 14:988-995.

Boeckmann B, Bairoch A, Apweiler R, Blatter M-C, Estreicher A, Gasteiger E, Martin MJ, Michoud K, O'Donovan C, and Phan I. 2003. The SWISS-PROT protein knowledgebase and its supplement TrEMBL in 2003. Nucleic Acids Research 31:365-370.

Bolger AM, Lohse M, and Usadel B. 2014. Trimmomatic: a flexible trimmer for Illumina sequence data. Bioinformatics 30:2114-2120.

Bru C, Courcelle E, Carrère S, Beausse Y, Dalmar S, and Kahn D. 2005. The ProDom database of protein domain families: more emphasis on 3D. Nucleic Acids Research 33:D212D215.

Camacho C, Coulouris G, Avagyan V, Ma N, Papadopoulos J, Bealer K, and Madden TL. 2009. BLAST+: architecture and applications. BMC Bioinformatics 10:421.

Chang H, Cheng T, Wu Y, Hu W, Long R, Liu C, Zhao P, and Xia Q. 2015. Transcriptomic Analysis of the Anterior Silk Gland in the Domestic Silkworm (Bombyx mori) - Insight into the Mechanism of Silk Formation and Spinning. PloS one 10:e0139424.

Cheng Dao-Jun LZ-Q, Meng Meng, Peng Jian, Qian Wen-Liang, Kang Li-Xia, Xia Qing-You. 2014. Characterization of Cytochrome P450 Genes Involving in Ecdysteroidogenesis in Silkworm (Bombyx mori). Scientia Agricultura Sinica 47:594-604.

Cheng T, Fu B, Wu Y, Long R, Liu C, and Xia Q. 2015. Transcriptome sequencing and positive selected genes analysis of Bombyx mandarina. PloS one. p e0122837.

Cheng X, Hu J, Li J, Chen J, Wang H, Mao T, Xue B, and Li B. 2018. The silk gland damage and the transcriptional response to detoxifying enzymes-related genes of Bombyx mori under phoxim exposure. Chemosphere 209:964-971.

Daimon T, Kozaki T, Niwa R, Kobayashi I, Furuta K, Namiki T, Uchino K, Banno Y, Katsuma S, Tamura T, Mita K, Sezutsu H, Nakayama M, Itoyama K, Shimada T, and Shinoda T. 2012. Precocious Metamorphosis in the Juvenile Hormone-Deficient Mutant of the Silkworm, Bombyx mori. PLoS genetics 8:e1002486.

Edgar RC. 2004. MUSCLE: multiple sequence alignment with high accuracy and high throughput. Nucleic Acids Research 32:1792-1797.

Fang S-M, Hu B-L, Zhou Q-Z, Yu Q-Y, and Zhang Z. 2015. Comparative analysis of the silk gland transcriptomes between the domestic and wild silkworms. BMC Genomics 16:60.

Feyereisen R. 1999. INSECT P450 ENZYMES. Annual Review of Entomology 44:507-533. Feyereisen R. 2015. Insect P450 inhibitors and insecticides: challenges and opportunities. Pest 
437

438

439

440

441

442

443

444

445

446

447

448

449

450

451

452

453

454

455

456

457

458

459

460

461

462

463

464

465

466

467

468

469

470

471

472

473

474

475

476

477

Management Science 71:793-800.

Gel B, and Serra E. 2017. karyoploteR: an R/Bioconductor package to plot customizable genomes displaying arbitrary data. Bioinformatics 33:3088-3090.

Haft DH, Selengut JD, and White O. 2003. The TIGRFAMs database of protein families. Nucleic Acids Research 31:371-373.

Harris R. 2007. Improved Pairwise Alignment of Genomic DNA: The Pennsylvania State University.

Haas B. 2014. TransDecoder. Available at http://transdecoder.github.io/.

Hulo N, Bairoch A, Bulliard V, Cerutti L, De Castro E, Langendijk-Genevaux PS, Pagni M, and Sigrist CJ. 2006. The PROSITE database. Nucleic Acids Research 34:D227-D230.

International Silkworm Genome C. 2008. The genome of a lepidopteran model insect, the silkworm Bombyx mori. Insect Biochem Mol Biol 38:1036-1045.

Kawamoto M. 2017. SilkBase v2.1. Available at http://silkbase.ab.a.u-tokyo.ac.jp/cgibin/blast.cgi? taxo name=Bombyx mandarina (accessed 20201020 2020).

Kawamoto M, Jouraku A, Toyoda A, Yokoi K, Minakuchi Y, Katsuma S, Fujiyama A, Kiuchi T, Yamamoto K, and Shimada T. 2019. High-quality genome assembly of the silkworm, Bombyx mori. Insect Biochem Mol Biol 107:53-62.

Kim D, Langmead B, and Salzberg SL. 2015. HISAT: a fast spliced aligner with low memory requirements. Nature Methods 12:357-360.

Krzywinski M, Schein J, Birol I, Connors J, Gascoyne R, Horsman D, Jones SJ, and Marra MA. 2009. Circos: an information aesthetic for comparative genomics. Genome Research 19:1639-1645.

Letunic I, and Bork P. 2019. Interactive Tree Of Life (iTOL) v4: recent updates and new developments. Nucleic Acids Research 47:W256-W259.

Mi H, Lazareva-Ulitsky B, Loo R, Kejariwal A, Vandergriff J, Rabkin S, Guo N, Muruganujan A, Doremieux O, and Campbell MJ. 2005. The PANTHER database of protein families, subfamilies, functions and pathways. Nucleic Acids Research 33:D284-D288.

Nebert DW, and Gonzalez FJ. 1987. P450 genes: structure, evolution, and regulation. Annu Rev Biochem 56:945-993.

Nelson DR. 1999. Cytochrome P450 and the individuality of species. Arch Biochem Biophys 369:1-10.

Pertea M, Pertea G, Antonescu C, Chang T-C, Mendell J, and Salzberg S. 2015. StringTie enables improved reconstruction of a transcriptome from RNA-seq reads. Nature Biotechnology 33.

Ponting CP, Schultz J, Milpetz F, and Bork P. 1999. SMART: identification and annotation of domains from signalling and extracellular protein sequences. Nucleic Acids Research 27:229-232.

Quevillon E, Silventoinen V, Pillai S, Harte N, Mulder N, Apweiler R, and Lopez R. 2005. InterProScan: protein domains identifier. Nucleic Acids Research 33:W116-120.

Robinson MD, McCarthy DJ, and Smyth GK. 2010. edgeR: a Bioconductor package for differential expression analysis of digital gene expression data. Bioinformatics 26:139- 
478

479

480

481

482

483

484

485

486

487

488

489

490

491

492

493

494

495

496

497

498

499

500

501

502

503

504

505

506

507

508

509

510

511

140.

Scott JG. 1999. Cytochromes P450 and insecticide resistance. Insect Biochem Mol Biol 29:757777.

Scott JG, Liu N, and Wen Z. 1998. Insect cytochromes P450: diversity, insecticide resistance and tolerance to plant toxins. Comp Biochem Physiol C Pharmacol Toxicol Endocrinol 121:147-155.

Stamatakis A. 2014. RAxML version 8: a tool for phylogenetic analysis and post-analysis of large phylogenies. Bioinformatics (Oxford, England) 30:1312-1313.

Wang H, Shi Y, Wang L, Liu S, Wu S, Yang Y, Feyereisen R, and Wu Y. 2018. CYP6AE gene cluster knockout in Helicoverpa armigera reveals role in detoxification of phytochemicals and insecticides. Nature Communications 9:4820.

Wang Y, Tang H, DeBarry JD, Tan X, Li J, Wang X, Lee T-h, Jin H, Marler B, and Guo H. 2012. MCScanX: a toolkit for detection and evolutionary analysis of gene synteny and collinearity. Nucleic Acids Research 40:e49-e49.

Xia Q, Zhou Z, Lu C, Cheng D, Dai F, Li B, Zhao P, Zha X, Cheng T, Chai C, Pan G, Xu J, Liu C, Lin Y, Qian J, Hou Y, Wu Z, Li G, Pan M, Li C, Shen Y, Lan X, Yuan L, Li T, Xu H, Yang G, Wan Y, Zhu Y, Yu M, Shen W, Wu D, Xiang Z, Yu J, Wang J, Li R, Shi J, Li H, Li G, Su J, Wang X, Li G, Zhang Z, Wu Q, Li J, Zhang Q, Wei N, Xu J, Sun H, Dong L, Liu D, Zhao S, Zhao X, Meng Q, Lan F, Huang X, Li Y, Fang L, Li C, Li D, Sun Y, Zhang Z, Yang Z, Huang Y, Xi Y, Qi Q, He D, Huang H, Zhang X, Wang Z, Li W, Cao Y, Yu Y, Yu H, Li J, Ye J, Chen H, Zhou Y, Liu B, Wang J, Ye J, Ji H, Li S, Ni P, Zhang J, Zhang Y, Zheng H, Mao B, Wang W, Ye C, Li S, Wang J, Wong GK, Yang H, and Biology Analysis G. 2004. A draft sequence for the genome of the domesticated silkworm (Bombyx mori). Science 306:1937-1940.

Xiang H, Liu X, Li M, Zhu Y, Wang L, Cui Y, Liu L, Fang G, Qian H, Xu A, Wang W, and Zhan S. 2018. The evolutionary road from wild moth to domestic silkworm. Nat Ecol Evol 2:1268-1279.

Xie C, Huang J, Ding Y, Wu J, Dong S, Kong L, Gao G, Li C-Y, and Wei L. 2011. KOBAS 2.0: A web server for annotation and identification of enriched pathways and diseases. Nucleic Acids Research 39:W316-322.

Yu L, Tang W, He W, Ma X, Vasseur L, Baxter SW, Yang G, Huang S, Song F, and You M. 2015. Characterization and expression of the cytochrome P450 gene family in diamondback moth, Plutella xylostella (L.). Sci Rep 5:8952. 


\section{Table $\mathbf{1}$ (on next page)}

Cytochrome P450 genes in B. mandarina and B. mori.

For $B$. mori, genes marked with ' $*$ ' and ' $\$$ ' have two counterparts in $B$. mandarina, and genes with ' $M$ ' mean manually modified by Kawamoto et al. (Kawamoto et al. 2019). For $B$. mandarina, genes with bold font involves in the KEGG map00981 (Insect hormone biosynthesis), and their amino acid (AA) length, exon number and protein sequence identity to $B$. mori homologs are listed in the last three column. 


\begin{tabular}{|c|c|c|c|c|c|}
\hline B. mandarina & B. mori & gene & $\begin{array}{c}\text { AA } \\
\text { length }\end{array}$ & $\begin{array}{l}\text { Exon } \\
\text { number }\end{array}$ & $\begin{array}{c}\text { Identity } \\
(\%)\end{array}$ \\
\hline \multicolumn{6}{|l|}{ CYP2Clan } \\
\hline XP_028039023.1 & KWMTBOMO06658 & CYP15C1 & 390 & 8 & 98.71 \\
\hline XP_028040961.1 & KWMTBOMO05795 & CYP18A1 & 541 & 7 & 100.00 \\
\hline XP_028040963.1 & KWMTBOMO05796 & CYP18B1 & 533 & 7 & 99.15 \\
\hline XP_028034668.1 & KWMTВOMO00033 & CYP303A1 & 498 & 3 & 99.00 \\
\hline MSTRG.449.1 & KWMTВОМО00224 & CYP305B1 & 487 & 9 & 100.00 \\
\hline XP_028040956.1 & KWMTBOMO05794 & CYP306A1 & 538 & 8 & 100.00 \\
\hline XP_028041590.1 & КWMTВОМO06147 & CYP307A1 & 536 & 2 & 99.51 \\
\hline \multicolumn{6}{|l|}{ CYP3Clan } \\
\hline XP_028040226.1 & KWMTBOMO12795 & CYP324A1 & 499 & 9 & 99.60 \\
\hline XP_028035792.1 & KWMTВOMO08858 & CYP332A1 & 500 & 8 & 99.58 \\
\hline XP_028039903.1 & KWMTВOМO09327 & CYP337A2 & 488 & 2 & 98.98 \\
\hline XP_028039890.1 & КWMTВОМО09329 & CYP337A1 & 489 & 2 & 98.57 \\
\hline XP_028030266.1 & KWMTВOMO07090 & CYP338A1 & 464 & 2 & 98.28 \\
\hline XP_028037108.1 & KWMTBOMO04531 & CYP354A1 & 516 & 9 & 99.03 \\
\hline- & KWMTBOMO12722 & CYP365A1 & - & - & - \\
\hline XP_028030488.1 & KWMTBOMO06852 & CYP6AB5 & 513 & 2 & 98.44 \\
\hline MSTRG.11650.2 & KWMTBOMO12342 & CYP6AB4 & 521 & 2 & 91.77 \\
\hline- & KWMTBOMO12343 & CYP6AB8 & - & - & - \\
\hline XP_028037754.1 & KWMTBOMO07237 & CYP6AE9 & 517 & 2 & 97.87 \\
\hline XP_028026771.1 & KWMTBOMO09944* & CYP6AE7 & 515 & 2 & 97.67 \\
\hline XP_028026792.1 & KWMTBOMO09944* & CYP6AE7 & 523 & 2 & 85.24 \\
\hline XP_028026744.1 & KWMTВOMO09945 & CYP6AE6P & 515 & 4 & 100.00 \\
\hline- & KWMTВOМO09947 & CYP6AE5 & - & - & - \\
\hline- & KWMTBOMO09950 & CYP6AE5 & - & - & - \\
\hline XP_028026755.1 & KWMTВOMO09951-1M & CYP6AE4 & 523 & 2 & 94.22 \\
\hline XP_028026857.1 & KWMTВOMO09951-2M & CYP6AE2 & 523 & 2 & 99.04 \\
\hline XP_028026790.1 & KWMTBOMO09952\$ & CYP6AE3P & 523 & 2 & 96.56 \\
\hline XP_028026883.1 & KWMTBOMO09952\$ & CYP6AE3P & 523 & 2 & 96.56 \\
\hline XP_028033575.1 & KWMTBOMO13805 & CYP6AE22 & 516 & 2 & 99.81 \\
\hline MSTRG.12374.1 & KWMTBOMO13412 & CYP6AN2 & 515 & 3 & 99.21 \\
\hline XP_028037243.1 & KWMTBOMO12654 & CYP6AU1 & 496 & 2 & 99.80 \\
\hline CYP6AV1-Bman & CYP6AV1 & CYP6AV1 & 500 & 2 & 99.800 \\
\hline- & KWMTBOMO05640 & CYP6AW1 & - & - & -- \\
\hline XP_028027733.1 & KWMTBOMO12622 & CYP6B29 & 505 & 2 & 100.00 \\
\hline XP_028036989.1 & KWMTBOMO10620 & CYP9A20 & 531 & 10 & 98.87 \\
\hline XP_028036963.1 & KWMTBOMO10621-1M & CYP9A19 & 490 & 10 & 90.67 \\
\hline XP_028042624.1 & KWMTBOMO10621-2M & CYP9A21 & 158 & 3 & 83.70 \\
\hline XP_028036964.1 & KWMTBOMO10626 & CYP9A22 & 531 & 11 & 98.87 \\
\hline
\end{tabular}




\begin{tabular}{|c|c|c|c|c|c|}
\hline XP_028033856.1 & KWMTBOMO10600 & CYP9AJ1 & 505 & 10 & 98.01 \\
\hline XP_028035894.1 & KWMTBOMO09346 & CYP9G1 & 495 & 9 & 92.53 \\
\hline XP_028036982.1 & КWMTВОМО10603 & CYP9G3 & 525 & 10 & 99.43 \\
\hline \multicolumn{6}{|l|}{ CYP4Clan } \\
\hline- & KWMTBOMO15697 & CYP340A1 & - & - & . \\
\hline- & KWMTВOMO15698 & CYP340A5P & - & - & - \\
\hline XP_028040572.1 & KWMTBOMO15699 & CYP340A4 & 488 & 10 & 97.10 \\
\hline XP_028040567.1 & KWMTBOMO15700 & CYP340A2 & 460 & 9 & 95.38 \\
\hline- & KWMTBOMO15704-15705M & CYP340A6 & - & - & - \\
\hline XP_028040566.1 & KWMTBOMO15707 & CYP340A3 & 489 & 10 & 99.80 \\
\hline XP_028043542.1 & KWMTBOMO15837 & CYP340B1 & 484 & 10 & 96.23 \\
\hline XP_028043540.1 & KWMTBOMO15838 & CYP340C1 & 495 & 11 & 99.02 \\
\hline XP_028040570.1 & KWMTBOMO15694 & CYP340D1 & 487 & 10 & 100.00 \\
\hline- & KWMTBOMO15695 & CYP340E1 & - & - & - \\
\hline XP_028031815.1 & KWMTBOMO15685 & CYP340F1 & 491 & 10 & 98.45 \\
\hline XP_028043541.1 & KWMTBOMO15835 & CYP340un1 & 487 & 12 & 97.94 \\
\hline- & CYP341A6 & CYP341A6 & - & - & - \\
\hline XP_028036361.1 & KWMTBOMO13274-13275M & CYP341A1 & 508 & 10 & 98.23 \\
\hline XP_028036360.1 & KWMTBOMO13276 & CYP341A3 & 504 & 10 & 94.82 \\
\hline XP_028036359.1 & KWMTBOMO13278 & CYP341A4 & 508 & 10 & 85.32 \\
\hline XP_028036358.1 & KWMTBOMO13279 & CYP341A5 & 508 & 10 & 99.16 \\
\hline XP_028036416.1 & KWMTBOMO13280 & CYP341A7 & 379 & 8 & 98.33 \\
\hline XP_028040208.1 & KWMTBOMO13324 & CYP341B1 & 512 & 10 & 99.34 \\
\hline XP_028026371.1 & KWMTBOMO13451 & CYP341C1 & 505 & 10 & 99.51 \\
\hline MSTRG.17906.1 & KWMTBOMO01080 & CYP366A1 & 557 & 11 & 99.55 \\
\hline- & KWMTВOMO09792 & CYP367A1 & - & - & - \\
\hline XP_028036546.1 & KWMTВOМO09791 & CYP367B1 & 497 & 10 & 96.58 \\
\hline XP_028041506.1 & KWMTВOMO01330 & CYP4AU2 & 495 & 10 & 99.09 \\
\hline- & KWMTBOMO12747 & CYP4AX1 & - & - & - \\
\hline- & KWMTBOMO12748 & CYP4AX2 & - & - & - \\
\hline XP_028042103.1 & KWMTBOMO07943 & CYP4G22 & 556 & 9 & 100.00 \\
\hline XP_028033176.1 & KWMTВОМО07978 & CYP4G24 & 562 & 11 & 94.61 \\
\hline XP_028033210.1 & KWMTBOMO07979-1M & CYP4G23 & 562 & 10 & 99.47 \\
\hline- & KWMTBOMO07979-2M & CYP4G23 & - & - & - \\
\hline XP_028028458.1 & KWMTBOMO07690 & CYP4L6 & 499 & 11 & 98.33 \\
\hline- & KWMTBOMO02817 & CYP4M9 & - & - & - \\
\hline XP_028040841.1 & KWMTВOMO02818 & CYP4M5 & 503 & 9 & 99.80 \\
\hline XP_028042717.1 & KWMTBOMO12746 & CYP4S5 & 125 & 1 & 100.00 \\
\hline- & KWMTBOMO12749 & CYP4S6 & - & - & - \\
\hline \multicolumn{6}{|l|}{ Mito.Clan } \\
\hline XP_028034514.1 & KWMTBOMO08259 & CYP301A1 & 528 & 8 & 100.00 \\
\hline
\end{tabular}




\begin{tabular}{lllrrr} 
XP_028032374.1 & KWMTBOMO13168 & CYP302A1 & 517 & 8 & 98.45 \\
XP_028030999.1 & KWMTBOMO03959-03960M & CYP314A1 & 516 & 9 & 92.49 \\
XP_028033300.1 & KWMTBOMO04611 & CYP315A1 & 496 & 8 & 99.58 \\
XP_028042905.1 & KWMTBOMO04516 & CYP333A2 & 420 & 9 & 98.81 \\
XP_028028513.1 & KWMTBOMO07693 & CYP333B2 & 510 & 10 & 99.22 \\
XP_028028461.1 & KWMTBOMO07694 & CYP333B1 & 512 & 10 & 99.41 \\
- & KWMTBOMO04339 & CYP333un1 & - & - & - \\
XP_028043257.1 & KWMTBOMO11023-11024M & CYP339A1 & 577 & 10 & 99.67 \\
XP_028042777.1 & KWMTBOMO08262 & CYP49A1 & 424 & 10 & 99.37 \\
- & KWMTBOMO11585 & CYP49A2 & - & - & - \\
\hline
\end{tabular}

1 


\section{Figure 1}

Synteny between $B$. mandarina and $B$. mori.

The left 28 chromosomes are from the $B$. mandarina genome and the right 28 chromosomes are from the $B$. mori genome. Echo chromosome number is marked on the karyotype bar on the circle, and ticks on each bar are the physical positions (unit: Mbp). Links are the homologous gene pairs identified by MCScanX and colored using the B. mori chromosome color scheme. 


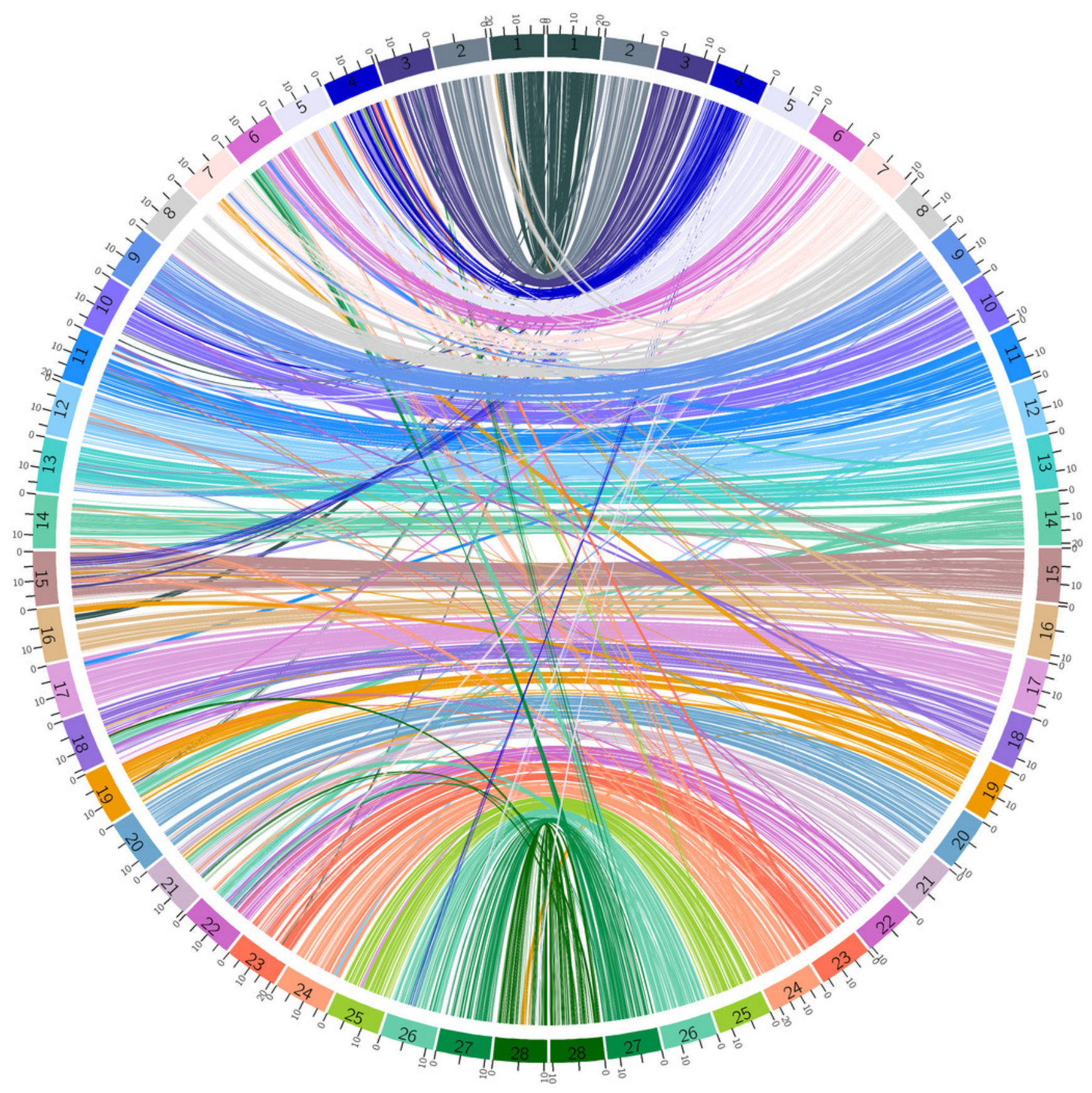


Figure 2

Phylogenetic tree of the cytochrome P450 genes in the wild and domestic silkworms.

The bootstrap value for 100 trials is labeled on each branch (only values $\geq 75$ are shown). The families attributed to insect P450 genes are marked for each clan on the circle. 


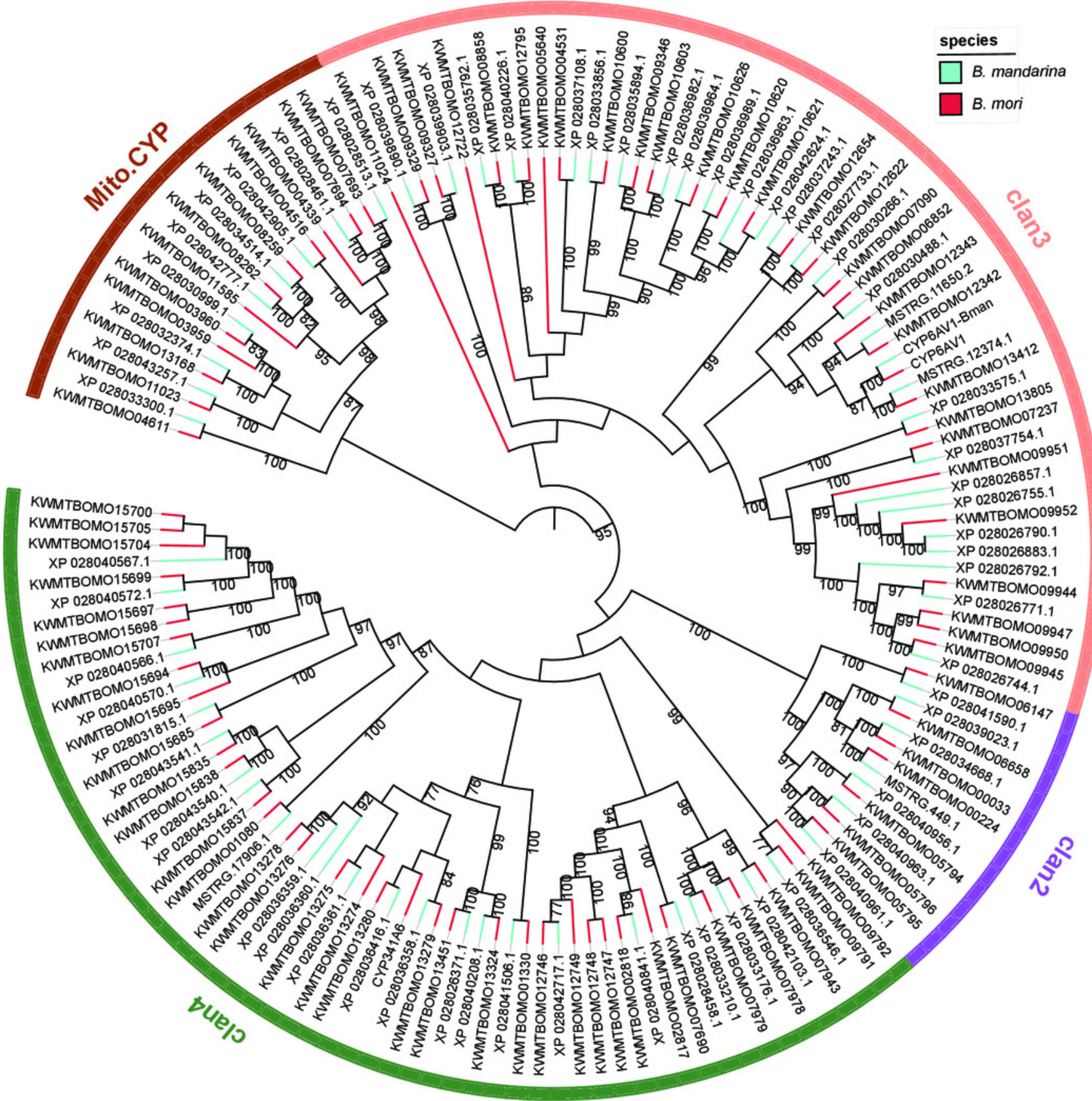




\section{Figure 3}

Physical locations of B. mandarina (A) and B. mori (B) P450 genes on the chromosomes.

Chromosomes are grey rectangles, bold number marked on each chromosome is their chromosome number, and ticks on each chromosome are the physical positions (unit: Mbp). P450 genes are colored according their clan group information. 
A<smiles>[TeH][TeH]</smiles>


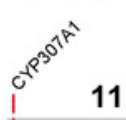
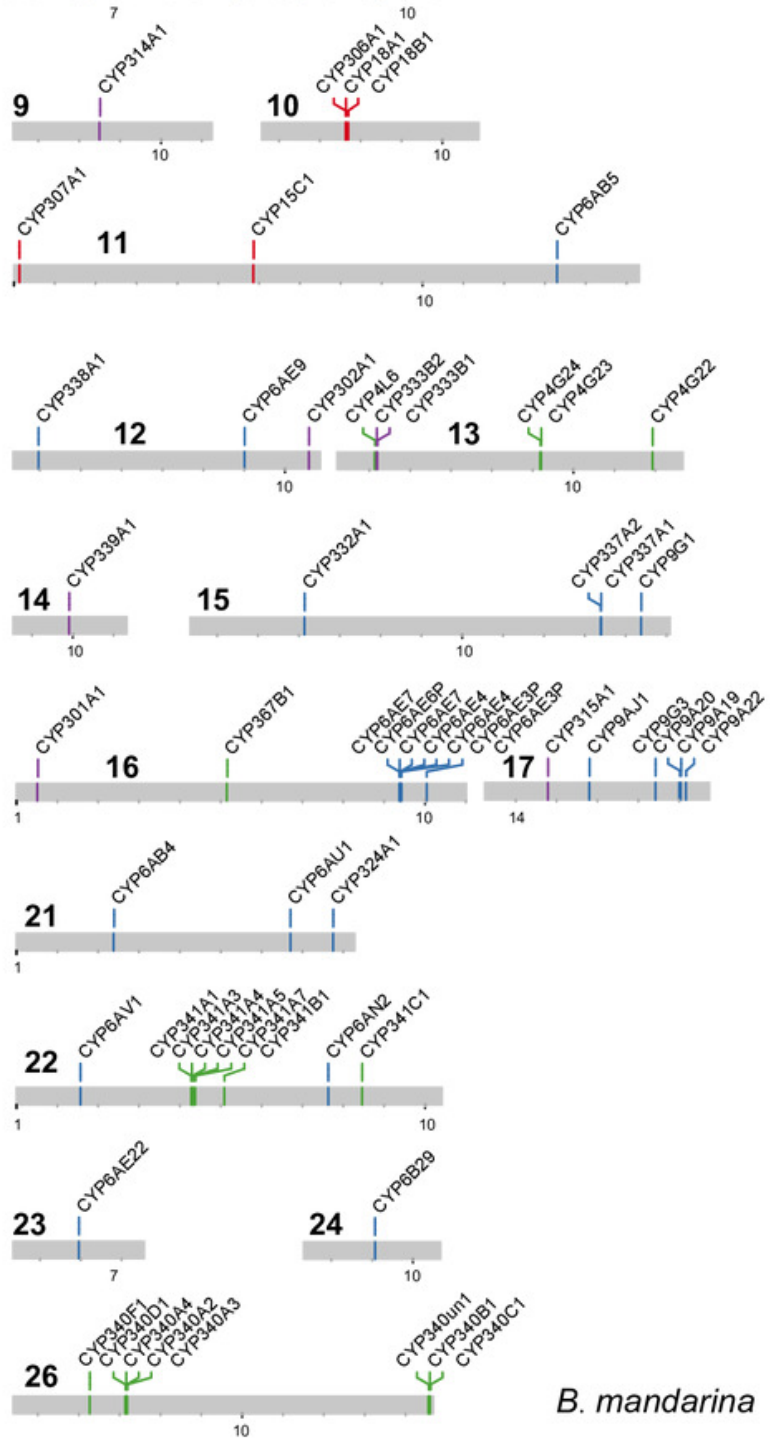

B. mandarina
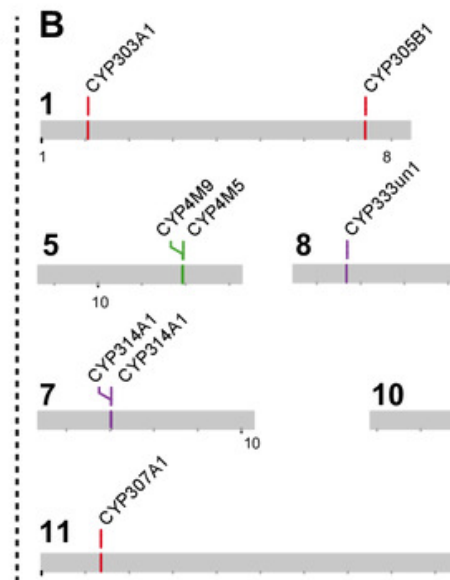

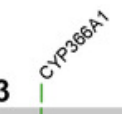
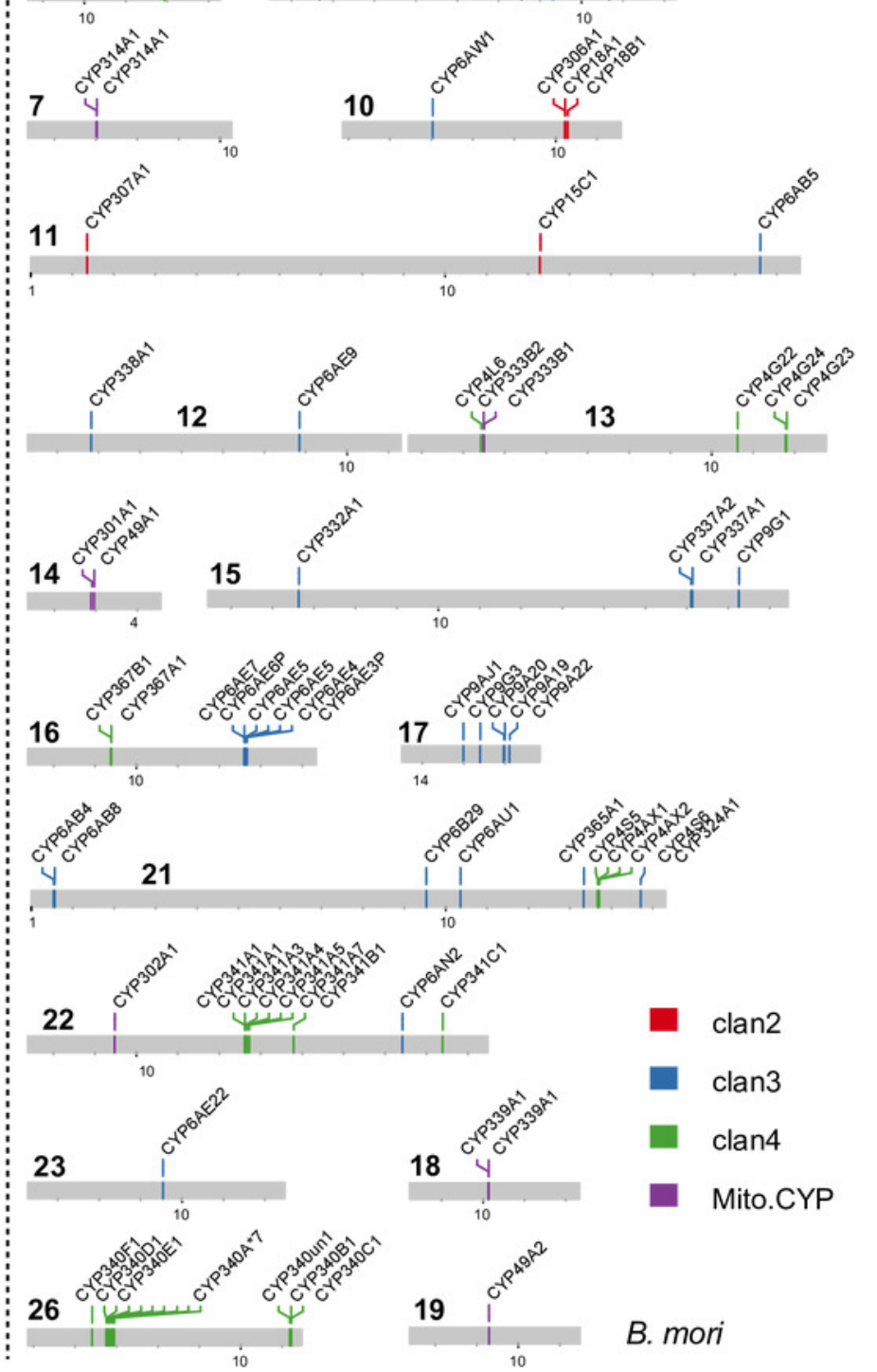


\section{Figure 4}

B. mandarina cytochrome P450 genes were significantly enriched in the insect hormone biosynthesis pathway.

This diagram of insect hormone biosynthesis pathway is adapted from KEGG (map00981), and the boxes colored with yolk yellow are the cytochrome P450 genes found in $B$. mandarina. FPPP, farnesyl diphosphate phosphatase; FOHSDR, NADP+-dependent farnesol dehydrogenase; ALDH, aldehyde dehydrogenase (NAD+); FAMeT, farsoic acid methyltransferase; JHAMT, juvenile hormone-III synthase; JHE, juvenile-hormone esterase; JHEH, juvenile hormone epoxide hydrolase; JHDK, juvenile hormone diol kinase; NvD, cholesterol 7-desaturase. 


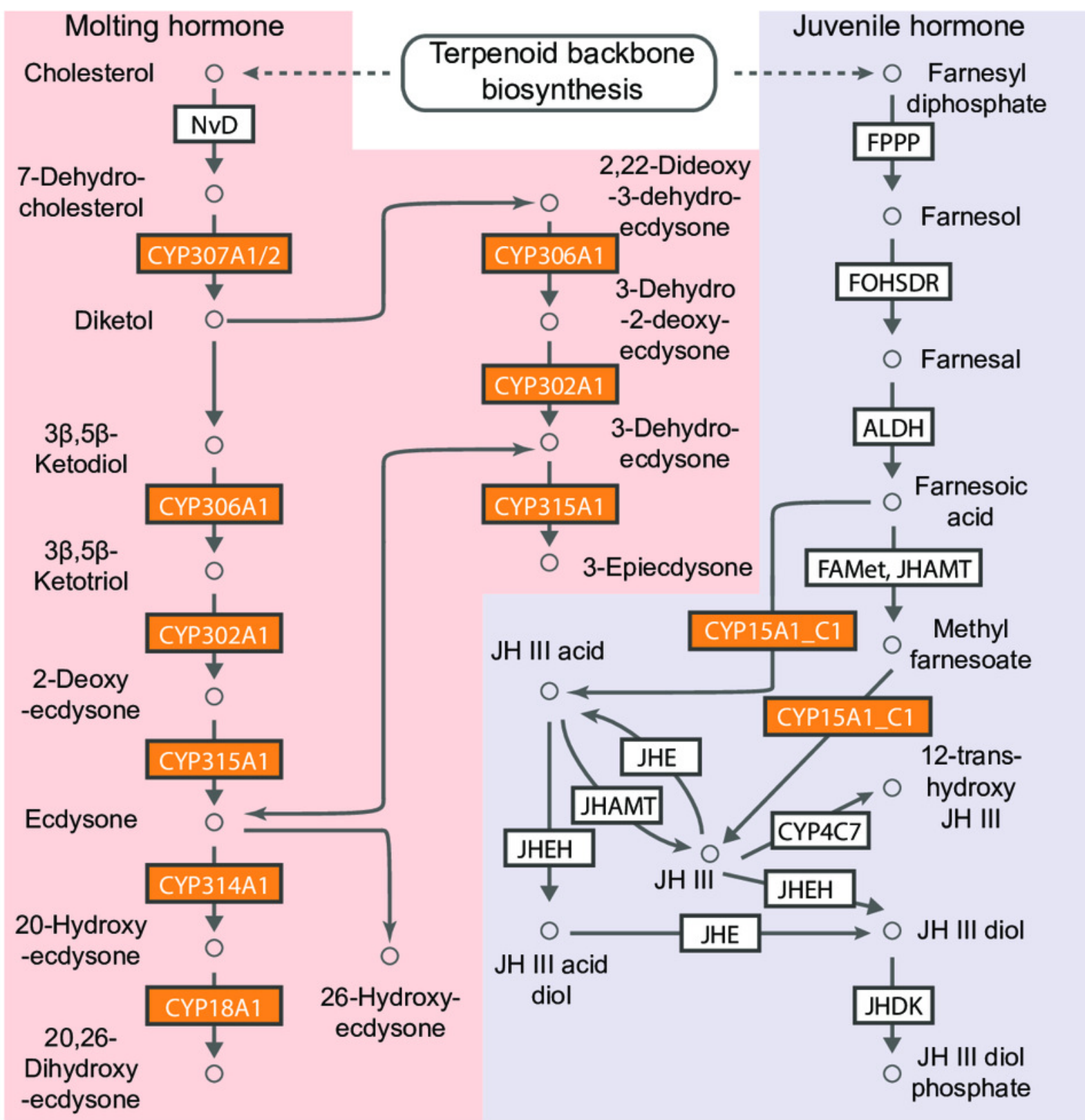




\section{Figure 5}

Expression analysis of cytochrome P450 genes across different tissues in B. mori (A) and B. mandarina (B).

The first column is the log10-transformed TMM (trimmed mean of M-values)-normalized expression data, and the tissue types are marked at the top of the heatmap. The second column is the - $\log 10$-transformed false discovery rate (FDR) value of DEG analyses from edgeR, and the comparaison scheme are also marked at the top of the heatmap. NS: not significant, namely, FDR > 0.05. Gene ID in Bold style is the CYP306A1. 
A



B.mori

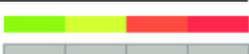

\begin{tabular}{|l|l|l|l|}
\hline KWMTBOMO04611 & KW \\
\hline
\end{tabular} КWMTBOMO11023 КWMTBOMO13168 KWMTBM KWMTBOMO03959 КWМтВОМО03960 KWMTBOMO11585 КWMTBOMO08262 КWMTBOMO08259 КWMTBOMO04516 KWMTBOMO04339 KWMTBOMO07694 KWMTBOMO07694 KWMTBOMO07693 KWMTBOMO11024 КWMTBOMO09329 КWMTBOMO09327 KWMTBOMO12722 KWMTBOMO08858 KWMTBOMO12795 КWMTВOMO12795 КWМтВОМО05640 KWMTBOMO04531
KWMTBOMO10600 KWMTBOMO10600
KWMTBOMO09346 KWMTBOMO10603 KWMTBOMO10626 KWMTBOMO10620 KWMTBMO10620 KWMTBOMO1062 KWMTBOMO12654
КWMTBOMO12622 КWMTBOMO07090 КWMTBOMO06852 KWMTBOMO12343 KWMTBOMO12342 KWMTOMO12342 КWMTBOMO13412 KWMTBOMO13805 КWMTBOMO07237 КWМтВОМО09951 КWMTBOMO09952 KWMTBOMO09944 KWMTBOMO09947 KWMTBOMO09950 KWMTBOMO09950 KWMTBOMO09945
KWMTBOMO06147 KWMTBOMO06658 КWMTВОМО00033 KWMTBOMO00224 КWMTBOMO05794 KWMTBOMO05796 KWMTBOMO05795 КWMTBOMO09792 КWMTBOMO09791 КWMTBOMO07943 КWMTBOMO07978 КWMTBOMO07979 KWMTBOMO07690 KWMTBOMO02817 KWMTBOMO02818 KWMTBOMO02818
KWMTBOMO12747 КWMTBOMO12748 КWMTBOMO12749 KWMTBOMO12746 KWMTBOMO01330 KWMTBOMO01330 KWMTBOMO13324 КWMTBOMO13451 KWMTBOMO13279 KWMTBOMO13280 КWMTBOMO13274 KWMTBOMO13275 KWMTBOMO1327 КWМтВОМО13276 KWMTBOMO13278 КWMTBOMO01080 КWMTBOMO15837 KWMTBOMO15838 KWMTBOMO15835 KWMTBOMO15685 КWMTBOM015695 КWMTВOMO15695 KWMTBOMO15694 KWMTBOMO1570 КWMTВOMO15698 KWMTBOMO15697 KWMTBOMO15699 KWMTBOMO15704 KWMTBOMO15705 KWMTBOMO15705

$\frac{\pi}{0}$

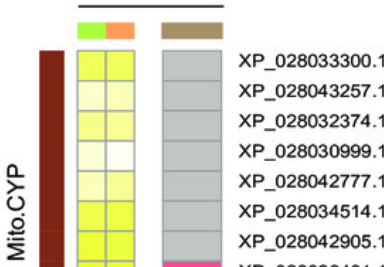

XP_028028461. XP_028028513.1

XP_028039890.1 XP_028039903.1 XP_028035792.1 XP_028040226.1 XP_028037108.1

XP_028033856.1 XP_028035894.1 XP_028036982.1 XP_028036964.1 XP_028036989.1 XP 028036963.1 XP_028042624.1 XP_028037243.1 XP_028027733.1 XP_028030266.1 XP_028030488.1 MSTRG.11650.2 CYP6AV1-Bman MSTRG.12374.1 XP_028033575.1 XP_028037754.1 XP_028026857.1 XP_028026755. 1 XP_028026790.1 XP_028026883.1 XP_028026792.1 XP_028026771.1 XP_028026744.1 XP_028041590.1 XP_028039023.1 XP_028034668.1 MSTRG.449.1

XP_028040956.1 XP_028040963.1 XP_028040961. XP_028036546.1 XP_028042103.1 XP_028033176.1 XP_028033210.1 XP_028028458.1 XP_028040841.1 XP 028042717.1 XP_028041506.1 XP_028040208.1 XP_028026371. XP_028036358.1 XP_028036416.1 XP_028036361. XP_028036360.1 XP_028036359.1 MSTRG.17906.1 XP_028043542.1 XP_028043540.1 XP_028043541.1 XP_028040570.1 XP_028031815.1 XP_028040566.1 XP_028040572.1 XP_028040567.1

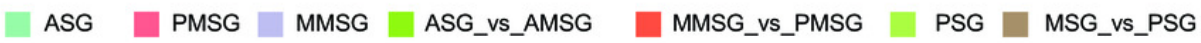
AMSG IPSG

$\begin{array}{lllllll}0 & 6 & 12 & -\lg (.05) & 2 & 5 & \text { NS }\end{array}$

Peer) reviewing PDF | (2020:06:49657:4:0:NEW 27 Dec 2020) 